\title{
26
}

\author{
DE \\ COLOPROCTOLOGIA
}

3

JULHO / SETEMBRO 2006

ARTIGOS ORIGINAIS

\section{Prevalência de Papilomavirus Humano (HPV) Anal, Genital e Oral, em Ambulatório Geral de Coloproctologia}

\section{Prevalence of Anal, Genital and Oral Human Papillomavirus (HPV) in General Ambulatory for Diseases of Colon and Rectum}

\author{
JOÃOCARLOS MAGI ${ }^{1}$; ELISA MARIADA SILVABRITO²; ELISABETETAEKO ONAGAGRECCO²; SONIAMARIA \\ MIRANDA PEREIRA ${ }^{3}$; GALDINO JOSÉ SITONIOFORMIGA ${ }^{4}$
}

${ }^{1}$ Médico do Serviço de Coloproctologia do Hospital Heliópolis-SP; ${ }^{2}$ Médica do Centro de Referencia e Treinamento do Programa Estadual de DST/AIDS-SP; ${ }^{3}$ Bióloga do Instituto Adolfo Lutz-SP;

${ }^{4}$ Chefe do Serviço de Coloproctologia do Hospital Heliópolis - SP - Brasil.

\begin{abstract}
MAGI JC; BRITO EMS; GRECCO ETO; PEREIRA SMM; FORMIGA GJS. Prevalência de Papilomavirus Humano (HPV) Anal, Genital e Oral, em Ambulatório Geral de Coloproctologia. Rev bras Coloproct, 2006;26(3): 233-238.

RESUMO: O Papilomavirus Humano (HPV) anal tem alta prevalência e incidência na população. O objetivo deste trabalho é verificar a prevalência de HPV anal, bucal e genital em ambulatório público de Coloproctologia, com base no exame de captura hibrida. Foram estudados 64 pacientes atendidos no ambulatório geral de Coloproctologia do Hospital Heliópolis, no período de 22/11/2005 a 20/12/2005, onde foram levantados, de forma prospectiva, dados clínicos, pessoais e comportamentais, identificando o perfil do grupo como não sendo de risco para HPV. Nenhum paciente deste grupo veio à consulta com queixa ou apresentando sinais de HPV ou outra doença sexualmente transmissível. Foram colhidos para cada paciente um esfregaço com escova de boca, outro de vulva/pênis e outro do ânus. O resultado mostrou que $15,62 \%$ dos pacientes apresentaram HPV anal, bucal ou genital, sendo que 4,68\% do total de pacientes apresentavam HPV anal, um paciente de alto risco associado ao HPV genital, outro misto e outro de baixo risco. Trinta por cento dos pacientes HPV + apresentavam dor anal como queixa principal. A conclusão é que a prevalência de HPV anal, genital e bucal é elevada, sendo que 4,68\% apresentavam HPV anal e daqueles HPV positivos $30 \%$ apresentavam dor anal na queixa principal.
\end{abstract}

Descritores: HPV anal, Papilomavirus Humano anal, incidência de HPV, dor anal-HPV, DST-HPV.

Trabalho realizado no Serviço de Coloproctologia do Hospital Heliópolis - São Paulo-SP; no Instituto Adolfo Lutz - São Paulo e no Centro de Referencia e Treinamento do Programa Estadual de DST/AIDS - São Paulo.-Brasil. 
Rev bras Coloproct

Julho/Setembro, 2006
Prevalência de Papilomavirus Humano (HPV) Anal, Genital e Oral, em Ambulatório Geral de Coloproctologia João Carlos Magi e Cols.
Vol. 26 $\mathbf{N}^{\circ} 3$

\section{Introdução}

O Papilomavirus Humano (HPV) é uma das Doenças Sexualmente Transmissíveis (DST) de maior incidência e prevalência no mundo, sendo comum o acometimento anal. ${ }^{1-10}$

O HPV anal predomina em alguns grupos, como: pacientes portadores de prurido anal idiopático, com antecedentes de tratamento da forma clinica de HPV anal, com antecedentes de tratamento de HPV genital, que procuram os ambulatórios de DST, HIV positivos, profissionais do sexo e com comportamento de risco para DST. Todavia, esta doença também acomete pessoas que não pertencem a estes grupos e pode ser adquirida de outras formas, além da relação sexual. ${ }^{6-14}$

Este é um trabalho prospectivo que tem o objetivo de verificar a prevalência de HPV anal, baseado no diagnóstico pelo exame de Captura Hibrida, em pacientes consultados em ambulatório de coloproctologia geral, onde foram levantados dados quanto à clinica, ao perfil sócio econômico e ao comportamento de risco de DST.

\section{Método}

Foram estudados de forma prospectiva 64 pacientes atendidos no ambulatório de coloproctologia geral do Serviço de Coloproctologia do Hospital Heliópolis São Paulo-SP no período de 22/11/2005 a 20/12/2005.

Este ambulatório é público e atende todos os pacientes com doenças coloproctológicas que procuram o serviço, sem seleção prévia. Após receberem o atendimento, alguns casos continuam o acompanhamento nos ambulatórios de coloproctologia de sub-especialidades, como câncer e doenças inflamatórias intestinais.

Todos os pacientes foram submetidos à colheita de Captura Hibrida com escova para teste de biologia molecular, usando-se o método de rotação, com um kit para pele perianal e canal anal, outro para boca, outro para vagina e para o pênis. O método de biologia molecular usado foi captura hibrida II. Foram levantados nos pacientes também dados clínicos, pessoais e comportamentais.

\section{Resultados}

De 64 pacientes estudados, 38 (59,37\%) eram do sexo masculino e 26 (40,63\%) feminino, com mé- dia de idade de 49,98 anos (23-82 anos). Trinta e três pacientes $(51,56 \%)$ eram de cor branca e $22(34,37 \%)$ pardos. Doze $(18,75 \%)$ pacientes nunca freqüentaram a escola, $19(29,68 \%)$ freqüentaram entre a primeira e quarta série e apenas um paciente $(1,5 \%)$ terminou um curso superior. Vinte e três pacientes $(35,93 \%)$ têm renda familiar menor que dois salários mínimos e 34 $(53,12 \%)$ têm renda entre dois e quatro salários mínimos. Trinta e oito pacientes $(59,37 \%)$ têm situação marital tipo união estável e nove $(14,06 \%)$ são viúvos.

A faixa etária de inicio das relações sexuais foi entre 13 e 15 anos em $25 \%$ dos pacientes, entre 16 e 19 anos em $44 \%$ e entre 20 e 25 anos em $25 \%$ dos pacientes. Treze pacientes $(20,31 \%)$ não tiveram relação sexual no último ano, quarenta e dois $(65,62 \%)$ tiveram apenas um parceiro sexual e apenas um paciente teve mais que cinco parceiros sexuais no último ano. Nos últimos cinco anos, onze pacientes (18\%) não tiveram relação sexual, trinta e cinco (57\%) tiveram apenas um parceiro sexual e três (5\%) tiveram mais de cinco parceiros sexuais. Quarenta e um pacientes $(64,06 \%)$ não têm parceiros eventuais, sendo que dez $(15,62 \%)$ pacientes que têm parceiros eventuais sempre usam preservativo nestas relações.

Apenas sete pacientes (10,93\%) sempre usam preservativo e nove $(14,06 \%)$ às vezes usam. Dos pacientes que usam preservativo, metade cobre com o preservativo apenas parte do pênis. Cinco $(7,81 \%)$ pacientes que usam preservativos colocam o preservativo no meio da relação ou quando ejaculam.

Apenas quinze pacientes $(23,43 \%)$ praticam sexo oral e destes três $(4,68 \%)$ usam preservativo para sexo oral. Quatorze pacientes $(21,87 \%)$ praticam sexo anal e apenas dois (3\%) usam preservativo.

Três $(4,68 \%)$ pacientes já fizeram uso de drogas injetáveis. Oito (12,5\%) pacientes já contraíram no passado alguma doença sexualmente transmissível, sendo que seis $(9,37 \%)$ em forma de corrimento e apenas um $(1,5 \%)$ em forma de verruga. Dezessete pacientes $(25 \%)$ já fizeram teste para HIV, sendo todos negativos. Seis pacientes $(9,37 \%)$ já tiveram câncer.

Em 37 pacientes $(57,81 \%)$, a queixa principal era relacionada à região anal, 22 (34\%) à região abdominal/intestinal, três $(4,77 \%)$ à região perineal $\mathrm{e}$ dois $(3,42 \%)$ não tinham sintomas. O exame retosigmoidoscópico foi normal em 21 pacientes $(32,81 \%)$; revelou patologias orificiais relacionadas com a queixa principal em $30(46,87 \%)$ pacientes e patolo- 
gias orificiais não relacionadas à queixa principal em $13(20,31 \%)$ pacientes.

Os resultados do exame de captura hibrida mostraram que um paciente tinha vírus HPV de alto risco no anus e na vulva, um paciente tinha vírus de baixo risco no anus, um paciente tinha vírus misto no anus, um paciente tinha vírus misto na vulva, um tinha vírus de alto risco na vulva, quatro pacientes tinham vírus de baixo risco na boca e um paciente tinha vírus misto no pênis. A idade média dos pacientes com HPV positivo nos três sítios é de 52,80 anos e destes com HPV anal positivo é 57,33 anos. A tabela 01 mostra o perfil dos pacientes com o vírus de HPV positivo, sendo sete mulheres e três homens, quatro com queixas abdominais, um encaminhado para biópsia de reto para pesquisa de Esquistossomose e sem queixas, um com queixa de dor anal, dois com queixas de dor anal associada a sangramento anal, um com queixa de prurido anal e um com queixa de prurido anal associado a sangramento anal. .

A tabela 02 relaciona os pacientes totais e os HPV positivos de acordo com a queixa principal e exame retosigmoidoscópico, indicando no grupo de HPV positivos os sítios acometidos e grupo de risco viral.

As tabelas mostram também que a prevalência de pacientes HPV positivos ligados a doenças orificiais foi $5 / 30(16,66 \%)$, sendo semelhante aos dos pacientes que procuraram o ambulatório por outros motivos coloproctológicos, 5/34 (14,7\%).

\section{Discussão}

A incidência e prevalência de HPV na população são elevadas. ${ }^{1-10}$

Alguns grupos como: pacientes com prurido anal idiopático, com antecedentes de tratamento de condiloma anal, antecedentes de tratamento de HPV genital, de ambulatórios de DST, HIV positivos, profissionais do sexo, com comportamento de risco para DST, têm maior incidência e prevalência para HPV. Todavia, a doença também incide em pessoas não pertencentes a estes grupos e pode ser adquirida por via não sexual. ${ }^{6-14}$

Este trabalho estuda a prevalência desta doença nos sítios mais comuns de acometimento, boca, genitais e ânus, em pessoas atendidas em ambulatório público de coloproctologia geral e que não pertencem aos grupos de maior incidência. 3,4,7,10,13

Notamos neste estudo que apenas um paciente tinha dois sítios acometidos (ânus e vulva), todos os outros apresentavam apenas um sítio acometido. Ocorre que o exame de captura híbrida tem sensibilidade aproximada de $90 \%$ e especificidade que na literatura varia de 90 a $50,9 \%$, sendo que os falsos negativos variam na literatura de 18,5 a $23 \%$ e os falsos positivos, segundo a literatura são aproximadamente $10,8 \%$, comparados aos exames de PCR e exame anátomopatológico. Apesar da maioria dos estudos se referir à região genital, Melbye et al ${ }^{27}$ afirmam que resultados similares aos genitais são obtidos na região anal. Os

\section{Tabela 1 - Perfil dos pacientes HPV positivos.}

\begin{tabular}{|c|c|c|c|c|c|}
\hline \multicolumn{2}{|c|}{ PacienteQueixa Principal } & Retosigmoidoscopia & \multirow{2}{*}{$\begin{array}{c}\text { Captura Hibrida }+ \\
\text { Boca }(\mathrm{br})\end{array}$} & \multirow{2}{*}{$\begin{array}{c}\text { idade } \\
65\end{array}$} & \multirow{2}{*}{$\frac{\text { sexo }}{\text { Fem }}$} \\
\hline 01 & Fechamento Colostomia & Normal & & & \\
\hline 02 & Prurido anal & Normal & Vulva-Anus (ar) & 51 & Fem \\
\hline 03 & Dor anal e sangramento & Hemorróidas & Anus (br) & 43 & Fem \\
\hline 04 & $\mathrm{~S} /$ queixas (biopsia retal p/ Esquistossomose) & Normal & Vulva (ar) & 50 & Fem \\
\hline 05 & Dificuldade para evacuar & Estenose anal-fibrose & Boca (br) & 80 & Masc \\
\hline 06 & Adenocarcinoma operado & Hemorróidas & Anus (ar e br) & 78 & Masc \\
\hline 07 & Dor anal e sangramento & $\begin{array}{l}\text { Fissura anal } \\
\text { com plicoma }\end{array}$ & Vulva (ar e br) & 38 & Fem \\
\hline 08 & Dor anal & $\begin{array}{l}\text { Plicoma anal e } \\
\text { fissura anal epitelizada }\end{array}$ & Boca (br) & 33 & Fem \\
\hline 09 & Adenocarcinoma operado & Normal & Boca (br) & 67 & Fem \\
\hline 10 & Prurido anal e sangramento anal & Hemorróidas & Pênis (ar e br) & 23 & Masc \\
\hline
\end{tabular}

Fem = feminino $; \quad$ Masc $=$ masculino $;$ br = baixo risco $;$ ar = alto risco 
Tabela 2 - Perfil clinico do número total de pacientes em relação ao perfil clinico, local de acometimento e risco viral dos pacientes $H P V$ positivos.

\begin{tabular}{lll}
\hline & Número total de pacientes/\% & Número de pacientes HPV +/\% / Local / Risco viral \\
\hline 1 & $08 / 12,50 \%$ & $1 / 1,54 \%$ / V e A (ar) \\
2 & $30 / 46,86 \%$ & $5 / 7,81 \%$ / A (ar); V (ar e br); 2 B (br); 1P (ar e br) \\
3 & $2 / 3,19 \%$ & $1 / 1,54 \%$ / V (ar) \\
4 & $11 / 17,13 \%$ & $2 / 3,19 \%$ / 2 B (br) \\
5 & $11 / 17,13 \%$ & $1 / 1,54 \%$ / A (ar) \\
6 & $2 / 3,19 \%$ & 0 \\
Total & $64 / 100 \%$ & $10 / 15,62 \%$ \\
\hline
\end{tabular}

1- queixa anal / perineal com retosigmoidoscopia normal; 2- queixa anal associada a lesão retosigmoidoscópica; 3- sem queixas e retosigmoidoscopia normal; 4- com queixas abdominais e sem queixas anais com retosigmoidoscopia normal; 5-com queixas abdominais elou sem queixas anais, retosigmoidoscopia com doença anal não associada; 6- queixa anal e retosigmoidoscopia com lesão sem relação com queixa anal.

trabalhos mostram também ser comum mais de um sitio de acometimento do HPV no mesmo paciente. Hernandez et al ${ }^{3}$ afirmam que a prevalência concomitante em mulheres saudáveis do HPV cervical e anal e entre vaginal e anal estão fortemente correlacionadas tanto a prevalência como a contaminação pela proximidade. Levando-se em conta estas informações e o resultado do estudo, é possível que os pacientes positivos tenham outros sítios de acometimento desta doença, como genital e anal. ${ }^{3,4,7,15-28}$

A prevalência de 15,62\% no grupo demonstra $\mathrm{o}$ aspecto endêmico da doença. Verificamos também o acometimento de pessoas com idade mais avançada (média de 52,80 anos) que deveriam ter maior resistência ao vírus. Devemos considerar também que a queda da incidência e prevalência com a idade, verificada no HPV genital, não ocorre necessariamente no HPV anal cuja incidência e prevalência podem se manter iguais. Estes dados associados às dificuldades de tratamento das formas sub-clinicas e latentes e a eficiência parcial do uso de preservativos para esta doença, demonstram a dificuldade de seu controle. Ultimamente estudos sobre vacinas dão esperanças no combate a esta doença. ${ }^{3,11,13,29-31}$

Verificamos também neste estudo que, dentre os pacientes HPV positivos, se destaca o sintoma de dor anal, levando-nos à reflexão quanto a possibilidade de dor anal idiopática ser considerada como mais um grupo de alta incidência de HPV na forma sub-clinica, mesmo porque o prurido anal pode ser considerado uma manifestação branda da dor anal, podendo ser ambos decorrentes de estimulo dos filetes nervosos locais. Um dos fatores que poderiam causar este estímulo seria a reação local ao HPV. Diante disto, consideramos a necessidade de estudos mais profundos relacionando o HPV com a dor anal idiopática. ${ }^{32-34}$

Este estudo mostra a prevalência de HPV em populações de baixo risco para esta doença e sugere a possibilidade de existir mais um grupo de alta incidência de HPV anal na forma sub-clinica e latente, a dor anal idiopática.

\section{Conclusões}

A prevalência de captura hibrida positiva para HPV anal, vulvar e oral, em pacientes apresentando baixo risco para HPV, foi de $15,62 \%$.

A prevalência de captura hibrida positiva para HPV anal neste grupo é de $4,68 \%$ ou $30 \%$ dos casos positivos, sendo um com tipo viral de alto risco de neoplasia apresentando HPV anal e vulvar positivo, um com tipo viral misto apenas anal e um com tipo viral de baixo risco apenas anal.

A prevalência de HPV positivo associado à dor anal como um dos sintomas foi de $4,68 \%$ ou $30 \%$ dos casos positivos. 
Rev bras Coloproct

Julho/Setembro, 2006
Prevalência de Papilomavirus Humano (HPV) Anal, Genital e Oral, em Ambulatório Geral de Coloproctologia João Carlos Magi e Cols.
Vol. 26

\begin{abstract}
Anal Human Papilomavirus (HPV) has a high prevalence and incidence in the population. The purpose of this project is to verify the prevalence of anal, oral and genital HPV at the Coloproctology Clinic, based on the hybrid capture exam. Sixty four patients were assisted at the clinic from November $22^{\text {nd }}$ to December $\mathbf{2 0}^{\text {th }} \mathbf{2 0 0 5}$. Clinical, personal and behavioral data of the patients were set up in a prospective way. The group profile was identified as not being a risky one to HPV. No patient of this group, when was assisted, had complained of or had presented any symptoms of HPV or any other sexually transmitted disease (STD). From each patient it was collected a mouth sample, a vulva/penis sample and an anus sample. The result indicated that of the $15,62 \%$ of the patients that had anal, oral, or genital HPV; 4,68\% of them presented anal HPV, one person with high risk of HPV to neoplasia had it associated to genital HPV; another one had a medium risk HPV and other a low risk one. The conclusion is that the prevalence of anal, genital and oral HPV was high, among this number, 4,68\% suffers from anal HPV and among HPV positive patient $30 \%$ had anal pain as the main complainty.
\end{abstract}

Key words: Anal HPV, Anal Human Papillomavirus, Incidence of HPV, Anal pain-HPV, STD-HPV.

\section{Referências}

1. Chuang TY, HO P, Kurland LT. Condylomata acuminatum in Rochester, Minn, 1950-1978. Epidemiology and Clinical Features. Arch Dermatol 1984;120: 469-75.

2. Luna GT. Epidemiology of Genital Human Papillomavirus. Gynecologic Cancer 1999;13:245-56.

3. Hernandez BY, McDuffie K, Zhu X. Anal Human Papillomavirus infection in Women and its Relationship with Cervical Infection. Câncer Epidemiol Biomarkers Prev 2005; 14(11):2550-60.

4. Smith EM, Ritchie JM, Yankowitz J. HPV prevalence and concordance in the cervix and oral cavity of pregnant women. Infect Dis Obstet Gynecol 2004;12(2):45-56.

5. Magi JC, Magi DAS, Reche LMC. Anuscopia com exacerbação para diagnóstico de Papillomavirus Humano Ano-Retal na forma Subclínica. Rev Bras Coloproct 2002; 22:178-83.

6. Magi JC, Rodrigues MRS, MorenoWD. A importância da anuscopia de alta resolução para o diagnóstico do papilomavirus humano anorretal na forma subclínica, das lesões anais intraepiteliais e do carcinoma "in situ" anal. Rev Col Bras Cir 2004; 31(1): 39-45.

7. Nadal SR, Manzione CR. Uso do colposcópio para avaliar a região perianal e o canal anal-padronização técnica da nomenclatura e indicações. Rev Bras Coloproct 2004; 24(4):379-81.

8. Nadal SR, Manzione CR, Galvão VM. Perianal diseases in HIV positive patients compared with seronegative population. Dis Colon Rectum 1999; 42: 649-54.

9. Nadal SR, Manzione CR. Infecção perianal recidivante pelo Papilomavirus humano. Rev Assoc Med Bras 2002; 48:14-5.

10. Patridge JM, Koutsky L. Genital Human Papillomavirus infection in men. Lancet Infect Dis 2006; 6: 21-31.

11. Chin-Hong PV, Vittinghoff E, Cranston RD. Age-Specific Prevalence of Anal Human Papillomavirus Infection in HIVnegative Sexually Active Men Who Have Sex with Men: The Explore Study. J Infect Dis 2004; 190: 2070-6.

12. Nadal SR, Manzione CR. Identificação dos grupos de risco para as doenças sexualmente transmissíveis. Rev Bras Coloproct 2003; 23:128-9.
13. Cañadas MP, Bosch FX, Junquera ML. Concordance of Prevalence of Human Papillomavirus DNA in Anogenital and Oral Infections in High-Risk Population. Journal of Clinical Microbiology 2004; 32 (3):1330-2.

14. Santos ALF, Derchain SFM, Calvert EB. Desempenho do exame colpocitológico com revisão por diferentes observadores e da captura hibrida II no diagnóstico da neoplasia intraepitelial cervical graus 2 e 3. Cad. Saúde Pública 2003; 19(4):639-44.

15. Cartle PE, Schiffman M, Burk RD. Restricted cross reactivity of hybrid capture 2 with nononcogenic human papillomavirus types. Cancer Epidemiol Biomarkers Prev 2002; 11(11): 1394-9.

16. Schiffman M, Herrero R, Hildeshein A. HPV DNA testing in cervical cancer screening: results from women in a high - risk province of Costa Rica. J Am Med Assoc 2000; 283:87-93.

17. Rebello G, Hallam N, Smart G. Human Papillomavirus testing and the management of women with mildly abnormal cervical smears: an observational study. BMJ 2001; 322:893-99.

18. Cox JT, Lorinczy AT, Schiffman MH. Human Papillomavirus testing Hybrid Capture appears to be useful in triaging women with a cytological diagnosis of ASCUS. Am J Obstet Gynecol 1995; 172: 946-54.

19. Clovel C, Masure M, Bory JP. Hybrid Capture II- based Human Papillomavirus detection a sensitive test to detect in routine high grade cervical lesions: a preliminary study on 1518 women. British J Cancer 1990; 80(9): 1306-11.

20. Clavel C, Massure M, Putaud I. Hybrid capture II, a new sensitive test for Human Papillomavirus detection. Comparison with hybrid capture I and PCR results in cervical lesions. J Clin Pathol 1998; 51: 737-40.

21. Bertagni A, Vagliasindi A, Ascari RA. Perianal Bowen's disease: a case report and review of the literature. Tumori 2003; 89(4): 16-8.

22. Peyton CL, Schiffman M, Lorincz AT. Comparison of PCR and Hybrid Capture based human detection systems using multiple cervical specimen coll. J Clin Microbiol 1998; 36(11): 3248-54. 
23. Kosel S, Burggraf S, Mommsen J. Type-specific detection of human papillomaviruses in a routine laboratory settingimproved sensitivity and specificity of PCR and sequence analysis compared to direct hybridization. Clin Chem Lab Med 2003; 41(6):787-91.

24. Lonky NM, Felix J, Tsadik GW. False negative hybrid capture II results related to altered adhesion molecule distribuition in women with atypical squamous cells pap smear results and tissue based human papillomavirus positive high grade cervical intraepithelial neoplasia. J Low Genit Tract Dis 2004; 8(4): 285-91.

25. Soderlund-Strand A, Rymark P, Andersson P. Comparison between the Hybrid Capture II test and a PCR based human papillomavirus detection method for diagnosis and posttreatment follow-up of cervical intraepithelial neoplasia. J Clin Microbiol 2005; 43(7):3260-6.

26. Kulmala SM, Syrjanen S, Shabalova I. Human papillomavirus testing with the hybrid capture 2 assay and PCR as screening tools. J Clin Microbiol 2004; 42(6): 2470-5.

27. Melbye M, Smith E, Wohlfahrt J. Anal and cervical abnormality in women prediction by human papillomavirus tests. Int J Cancer 1996; 68(5): 559-64.

28. Harper DM, Franco EL, Wheler C. Efficacy of a bivalent L1 virus-like porticle vaccine in prevention of infection with Human Papillomavirus types 16 and 18 in yong women: a randomized controlled trial. Lancet 2004; 364: 1757-65.
29. Galloway DA. Papillomavirus vaccines in clinical trials. Lancet Infect Dis 2003; 3: 469-75.

30. Villa LL, Costa RLR, Petta CA. Prophilactic quadrivalent Human Papillomavirus (types 6,11,16 and 18) L1 virus - like particle vaccine in young women: a randomised double - blind placebo - controlled multicentre phase II efficacy trial. Lancet 2005; 5:101-7.

31. Nadal SR. Eficácia dos preservativos na prevenção das DST. Rev Bras Coloproct 2003; 23:225-7.

32. Anand P. Capsaicin and menthol in the treatment of itch and pain: recently cloned receptors provide the key. Gut 2003; 52(9): 1233-5.

33. Vicent C. Anorectal pain and irritation: anal fissure, levator syndrome, proctalgia fugax and pruritus ani. Prim Cale 1999; 26(1):53-68.

34. Santos Junior JCM. Fissura anal. Rev Bras Coloproct 2001; 21(2):99-108.

\section{Endereço para correspondência:}

JOÃO CARLOS MAGI

Rua Paraíba, 717 - Centro.

09.521-070 -S. Caetano do Sul (SP) - Brasil

Tel/fax (11) 4221-4358 